\title{
Prevalência e fatores associados ao desmame precoce: uma revisão integrativa
}

\author{
Prevalence and factors associated with early weaning: an integrative review
}

Prevalencia y factores asociados al destete precoz: una revisión integradora

\begin{abstract}
RESUMO
Objetivo: Analisar a temática do aleitamento materno (AM), no que tange à importância da prática e à prevalência, destacando os fatores associados ao desmame precoce. Métodos: Trata-se de revisão integrativa de literatura, utilizando-se as bases de dados SciELO, Bireme, Google Scholar, LILACS e Medline, com os descritores breastfeeding, prevalence e weaning. Foram analisados trabalhos publicados nos idiomas português, inglês e espanhol no período entre 2009 e 2019. Resultados: a pesquisa, a partir de 29 trabalhos selecionados, apontou que há grandes variações regionais nas prevalências do AM e que a interrupção precoce pode estar associada às condições do meio e/ou da mãe; os conhecimentos, crenças e tabus maternos desempenham uma grande influência na continuidade da amamentação e no padrão de introdução alimentar da criança. Conclusão: faz-se necessário adequar e fortalecer a escuta profissional no sentido de prestar atendimento que acolha as dúvidas, os medos e as incertezas moldadas pelo ambiente no qual as gestantes e nutrizes estão inseridas.
\end{abstract}

DESCRITORES: Aleitamento Materno; Prevalência; Desmame.

\section{ABSTRACT}

Objective: To analyze the breastfeeding theme, especially regarding the practice importance and its prevalence, highlighting the factors associated with early weaning. Methods: Integrative literature review, using the databases SciELO, Bireme, Scholar Google, LILACS and Medline with the descriptors breastfeeding, prevalence and weaning. Papers in Portuguese, Spanish and English, published in the period from 2009 to 2019, were included. Results: The research, based on 29 works selected, pointed out that there are significant regional variances in the BF prevalence and that the early discontinuation may be associated with environmental conditions and/or related the mother; maternal knowledge, beliefs and taboos play a major role in the continuity of BF and in the child's dietary introduction pattern. Conclusion: It is necessary to adapt and strengthen professional listening in the sense of providing assistance that welcomes doubts, fears and uncertainties, shaped by the environment in which pregnant women and nursing mothers are inserted.

ESCRIPTORS: Breastfeeding; Prevalence; Weaning.

\section{RESUMEN}

Objetivo: Analizar la temática del amamantamiento, especialmente en lo que toca a la importancia de la práctica y su prevalencia, destacando los factores asociados al destete precoz. Método: Se trata de una revisión integradora de la literatura, utilizando las bases de datos SciELO, Bireme, Google Scholar, LILACS y Medline, con los descriptores: lactancia materna, prevalencia y destete. Se incluyeron trabajos en português, español y inglês, publicados entre 2009 y 2019. Resultados: La investigación, basada em 29 trabajos selecionados, señaló que la interrupción precoz de la LM exclusiva puede estar asociada con las condiciones del ambiente y/o de la madre; el conocimiento, las creencias y los tabúes maternos juegan un papel importante en la continuidad de la LM y em el patrón de introducción a la dieta del niño. Conclusión: Es necessário adaptar y fortalecer la escucha professional, en el sentido de brindar una atención que acoja dudas, miedos e incertidumbres, moldeado por el entorno en el que se insertan las mujeres embarazadas y las madres lactantes.

DESCRIPTORES: Lactancia Materna; Prevalencia; Destete.

RECEBIDO EM: 28/09/2020 APROVADO EM: 23/10/2020

\section{Thiana Pinho Araújo}

Nutricionista. Especialista em Nutrição Clínica: Metabolismo, Prática e Terapia Nutricional. Mestranda do Programa de Pós-Graduação Saúde, Ambiente e Sociedade na Amazônia do Instituto de Ciências da Saúde da Universidade Federal do Pará (ICS/UFPA). ORCID: 0000-0001-9384-8084 


\section{Lucrecia Aline Cabral Formigosa}

Enfermeira. Especialista em Saúde Coletiva. Mestranda do Programa de Pós-Graduação Saúde, Ambiente e Sociedade na Amazônia do Instituto de Ciências da Saúde da Universidade Federal do Pará (ICS/UFPA).

ORCID: 0000-0003-4245-672X

\section{Adrianne Pureza Maciel}

Nutricionista. Especialista em Saúde do Idoso e em Nutrição Clínica: Metabolismo, Prática e Terapia Nutricional. Nutricionista do Restaurante Universitário da Universidade Federal do Pará (RU/UFPA).

ORCID: 0000-0001-5546-2331

\section{INTRODUÇÃO}

0 $s$ primeiros anos de vida são marcados pelo rápido crescimento $\mathrm{e}$ desenvolvimento, tendo a alimentação um papel essencial, uma vez que a qualidade e a quantidade do que é consumido nessa fase têm repercussões ao longo da vida, associando-se ao perfil de saúde ${ }^{1}$.

Recomendado pela Organização Mundial da Saúde (OMS), o leite materno (LM) é o único alimento a ser ofertado no primeiro semestre de vida; depois, deve-se iniciar a alimentação complementar (AC), dando preferência a alimentos saudáveis, ofertados em refeições equilibradas em macro e micronutrientes ${ }^{2}$.

Ocorrendo de forma inadequada, a AC pode resultar em consequências danosas para a saúde, aumentando o risco de contaminação e de reações alérgicas, além de interferir na absorção de nutrientes do LM e predispor ao desmame precoce ${ }^{1}$.

Não obstante todas as evidências científicas comprovando a superioridade do aleitamento materno (AM) sobre outras formas de alimentar a criança pequena, a prática ainda não é exercida plenamente, sobretudo na sua forma exclusiva ${ }^{3}$.

Dados globais apontam prevalência de aleitamento materno exclusivo (AME) na faixa de 0 a 6 meses de cerca de 35\% enquanto dados nacionais da II Pesquisa de Prevalência de Aleitamento Materno nas Capitais e Distrito Federal apontaram $41 \%$ de $\mathrm{AME}^{2}$.

A necessidade do aumento das taxas de AME até os 6 meses de vida e AM complementada até os dois anos de vida sustenta-se com dados estimativos de que até 13\% de mortes de crianças menores de 5 anos em todo o mundo poderiam ser evitadas a partir destas práticas, haja vista a relação bem estabelecida entre o desmame precoce e as mortes por doença diarreica e infecções respiratórias especialmente entre crianças com menor nível socioeconômico. Em estudo realizado em 14 municípios da grande São Paulo, por exemplo, foi estimada influência do AM com o Coeficiente de Mortalidade Infantil em 9,3\% ${ }^{3}$.

A interrupção precoce do AME está intimamente associado às condições do meio e/ou relacionadas à mãe, os quais também exercem grande influência na introdução alimentar da criança ${ }^{4}$.

Desta forma, o estudo objetivou analisar as pesquisas desenvolvidas sobre a temática do $\mathrm{AM}$ no que tange à importância da prática e à sua prevalência, destacando os fatores relacionados com o desmame precoce, a fim de reforçar aos profissionais da área e à comunidade acerca do papel primordial que o ato de amamentar tem na prevenção da morbimortalidade materno-infantil.

\section{MÉTODO}

Trata-se de uma revisão integrativa da literatura, a qual possibilitou ampla abordagem metodológica através da compilação de informações atualizadas provenientes de estudos não experimentais ${ }^{5}$. Para tanto, a construção do trabalho seguiu as seis fases descritas a seguir:

1- Definição das questões norteadoras: qual a prevalência do aleitamento materno no Brasil e no mundo e quais os fatores associados ao desmame precoce?

2- Busca na literatura: a seleção dos estudos ocorreu no período de agosto/2019 a janeiro/2020, nas bases de dados SciELO, Bireme,
Google Scholar, LILACS e MEDLINE, utilizando-se os descritores DeCS breastfeeding, prevalence e weaning, juntamente com operador booleano and. Incluíram-se pesquisas publicadas entre 2009 e 2019 nos idiomas inglês, português e espanhol que abordassem a temática relacionada com a importância e a prevalência do $\mathrm{AM}$, bem como os fatores associados à manutenção e/ou abandono da prática. Procedeu-se a leitura do título e resumo dos trabalhos e excluíram-se materiais duplicados, com resultados inconclusivos $\mathrm{e}$ fora da temática escolhida.

3- Coleta de dados: procedeu-se a aplicação dos critérios de inclusão e exclusão, concomitante à leitura exploratória dos títulos e resumos selecionados a partir da busca em bases de dados e, após a triagem e seleção dos artigos por meio da aplicação do instrumento URSI adaptado para a pesquisa, uma dupla de pesquisadoras ficou responsável pela categorização de informações pertinentes em temas.

4- Análise: os artigos selecionados foram categorizados em quatro temas diretamente ligados às perguntas norteadoras: 1- Benefícios do LM e do ato de amamentar; 2- Prevalência da prática no Brasil e no mundo; 3- Fatores que interferem no processo de desmane precoce; $\mathrm{e}$ 4- Introdução alimentar precoce.

5- Discussão: a consolidação dos dados foi realizada a partir da reunião dos dados e discussão entre as autoras sobre os quatro temas-eixo para responder às questôes norteadoras. 
6- Apresentação: a redução dos achados, encontra-se exposta nas etapas a seguir.

\section{RESULTADOS}

Após, análise descritiva dos dados de todos os artigos obteve-se uma amostra de 29 trabalhos, nos idiomas português, inglês e espanhol, compatíveis com o tema proposto, os quais foram categorizados a fim de facilitar a discussão dos achados (Quadro 1).

\begin{tabular}{|c|c|c|c|}
\hline AUTORES & TítULO & PERIÓDICO & $\begin{array}{l}\text { ANO DE } \\
\text { PUBLICAÇÃO }\end{array}$ \\
\hline Costa et al. ${ }^{6}$ & $\begin{array}{l}\text { Promoção do aleitamento materno no contexto da Estratégia } \\
\text { de Saúde da Família. }\end{array}$ & $\begin{array}{l}\text { Revista Rede de Cuidados em } \\
\text { Saúde }\end{array}$ & 2019 \\
\hline Oliveira et al. ${ }^{7}$ & $\begin{array}{l}\text { Breastfeeding duration and associated factors between } \\
\qquad 1960 \text { and } 2000 .\end{array}$ & Jornal de Pediatria & 2017 \\
\hline Walters et al. ${ }^{8}$ & $\begin{array}{l}\text { The cost of not breastfeeding: global results from a new tool. } \\
\text { Health policy plan. }\end{array}$ & Health policy plan & 2019 \\
\hline Margotti et al. ${ }^{9}$ & $\begin{array}{l}\text { Fatores relacionados ao aleitamento materno exclusivo } \\
\text { em bebês nascidos em Hospital Amigo da Criança em uma } \\
\text { capital do Norte brasileiro. }\end{array}$ & Saúde Debate & 2017 \\
\hline José et al.10 & Relação entre desmame precoce e alergias alimentares. & Visão acadêmica. & 2016 \\
\hline $\begin{array}{l}\text { Carvalho-Ramos } \\
\text { et al. }{ }^{11}\end{array}$ & Breastfeeding increases microbial community resilience. & Jornal de Pediatria & 2018 \\
\hline Tessari et al. ${ }^{12}$ & $\begin{array}{l}\text { Percepção de mães e pais adolescentes sobre o aleitamento } \\
\text { materno. }\end{array}$ & Enfermagem em foco & 2019 \\
\hline $\begin{array}{l}\text { Del Ciampo \& Del } \\
\text { Ciampo }^{13}\end{array}$ & $\begin{array}{l}\begin{array}{l}\text { Breastfeeding and the benefits of lactation for women's } \\
\text { health. }\end{array} \\
\end{array}$ & $\begin{array}{l}\text { Revista Brasileira de Ginecologia } \\
\text { e Obstetrícia. }\end{array}$ & 2018 \\
\hline Santana et al..$^{14}$ & $\begin{array}{l}\text { Factors associated with breastfeeding maintenance for } 12 \\
\text { months or more: a systematic review. }\end{array}$ & Jornal de Pediatria & 2018 \\
\hline Nunes LM ${ }^{15}$ & Importância do aleitamento materno na atualidade & Boletim Científico de Pediatria & 2015 \\
\hline Rollings et al. ${ }^{16}$ & $\begin{array}{l}\text { Why invest, and what it will take to improve breastfeeding } \\
\text { practices? }\end{array}$ & The Lancet & 2016 \\
\hline Silva et al. ${ }^{17}$ & $\begin{array}{l}\text { Maternal breastfeeding: indicators and factors associated } \\
\text { with exclusive breastfeeding in a subnormal urban cluster } \\
\text { assisted by the Family Health Strategy. }\end{array}$ & Jornal de Pediatria & 2019 \\
\hline Victora et al. ${ }^{18}$ & $\begin{array}{l}\text { Breastfeeding in the 21st century: epidemiology, mechanis- } \\
\text { ms and lifelong effect. }\end{array}$ & The Lancet & 2016 \\
\hline Zielińska et al. ${ }^{19}$ & $\begin{array}{l}\text { Breastfeeding knowledge and exclusive breastfeeding of } \\
\text { infants in first six months of life. }\end{array}$ & $\begin{array}{c}\text { Roczniki Państwowego Zakładu } \\
\text { Higieny }\end{array}$ & 2017 \\
\hline Mosca et al..$^{20}$ & $\begin{array}{l}\text { Determinants of breastfeeding discontinuation in an Italian } \\
\text { cohort of mother-infant dyads in the first six months of life: a } \\
\text { randomized controlled trial. }\end{array}$ & Italian Journal of Pediatrics & 2018 \\
\hline $\begin{array}{l}\text { Ramiro González } \\
\text { et al. }{ }^{21}\end{array}$ & $\begin{array}{l}\text { Prevalencia de la lactancia materna y factores asociados con } \\
\text { el inicio y la duración de la lactancia materna exclusiva en la Co- } \\
\text { munidad de Madrid entre los participantes en el estudio ELOIN }\end{array}$ & Anales de Pediatría & 2018 \\
\hline Mota-Castillo et al. ${ }^{22}$ & $\begin{array}{l}\text { Experiencias y creencias de madre sobre la lactancia materna } \\
\text { exclusiva en una región de México. }\end{array}$ & Journal of Nursing and Health & 2019 \\
\hline McLennan ${ }^{23}$ & $\begin{array}{l}\text { Changes over time in early complementary feeding of breas- } \\
\text { tfeed infants on the island of Hispaniola }\end{array}$ & $\begin{array}{l}\text { Revista Panamericana de Salud } \\
\text { Publica }\end{array}$ & 2018 \\
\hline Boccolini et al. ${ }^{24}$ & $\begin{array}{l}\text { Tendência de indicadores do aleitamento materno no Brasil } \\
\text { em três décadas. }\end{array}$ & Revista de Saúde Pública & 2017 \\
\hline
\end{tabular}




\section{artigo}

Araújo, T.P.; Formigosa, L.A.C.; Maciel, A.P.

Prevalência e fatores associados ao desmame precoce: uma revisão integrativa

\begin{tabular}{|c|c|c|c|}
\hline Santos et al. ${ }^{25}$ & $\begin{array}{c}\text { Desmame precoce em crianças atendidas na Estratégia } \\
\text { Saúde da Família. }\end{array}$ & $\begin{array}{l}\text { Revista Eletrônica de Enferma- } \\
\text { gem }\end{array}$ & 2018 \\
\hline Monteiro et al. ${ }^{26}$ & Influence of maternity leave on exclusive breastfeeding. & Jornal de Pediatria & 2017 \\
\hline Schincaglia et al. ${ }^{27}$ & $\begin{array}{l}\text { Práticas alimentares e fatores associados à introdução pre- } \\
\text { coce da alimentação complementar entre crianças menores } \\
\text { de seis meses na região noroeste de Goiânia. }\end{array}$ & $\begin{array}{l}\text { Epidemiologia e Serviços de } \\
\text { Saúde }\end{array}$ & 2015 \\
\hline Mariano et al. ${ }^{28}$ & $\begin{array}{l}\text { Saberes maternos acerca da alimentação complementar. Cad } \\
\text { da Esc de Saúde. }\end{array}$ & Cadernos da Escola de Saúde & 2017 \\
\hline Mosquera et al. ${ }^{29}$ & $\begin{array}{l}\text { Factors affecting exclusive breastfeeding in the first month of } \\
\text { life among Amazonian children. }\end{array}$ & PLOS ONE & 2019 \\
\hline Mendes et al. ${ }^{30}$ & $\begin{array}{l}\text { Fatores relacionados com uma menor duração total do } \\
\text { aleitamento materno. }\end{array}$ & Ciência e Saúde Coletiva & 2018 \\
\hline Nóbrega et al. ${ }^{31}$ & $\begin{array}{l}\text { As redes sociais de apoio para o Aleitamento Materno: uma } \\
\text { pesquisa-ação. }\end{array}$ & Saúde Debate & 2019 \\
\hline Borges $^{32}$ & $\begin{array}{c}\text { Caracterização e prevalência do aleitamento materno em } \\
\text { uma população atendida na rede pública de saúde de Pal- } \\
\text { mas/TO, Brasil. }\end{array}$ & $\begin{array}{c}\text { Revista Brasileira de Pesquisa em } \\
\text { Saúde }\end{array}$ & 2016 \\
\hline Albuquerque et al. ${ }^{33}$ & $\begin{array}{l}\text { Hábitos alimentares de crianças com até } 6 \text { meses em ali- } \\
\text { mentação complementar e/ou desmame precoce. }\end{array}$ & Enfermagem atual. & 2018 \\
\hline Giesta et al. ${ }^{34}$ & $\begin{array}{l}\text { Fatores associados à introdução precoce de alimentos } \\
\text { ultraprocessados na alimentação de crianças menores de } \\
\text { dois anos. }\end{array}$ & Ciência e Saúde Coletiva & 2019 \\
\hline
\end{tabular}

\section{DISCUSSÃO}

\section{Benefícios do LM e do ato de ama- mentar}

Historicamente, a amamentação vem sendo incentivada e valorizada por organismos nacionais e internacionais como estratégia para redução da mortalidade materno-infantil6. Estima-se que a prática tenha o potencial de salvar mais de 800 mil vidas de crianças menores de 5 anos por ano no mundo 7 , além de prevenir 98.243 mortes de mulheres por câncer de mama e ovário e diabetes tipo II ao ano ${ }^{8}$.

Por seu fator imunoprotetor, o LM reduz a ocorrência de óbitos causados por doenças infectocontagiosas, bem como a frequência de internações hospitalares e as chances de desenvolver doenças crônicas, manifestações alérgicas e intolerância alimentar ${ }^{9,10,11}$.

No tocante à saúde da nutriz, a prática favorece a construção do vínculo afetivo entre o binômio mãe e filho; evita hemorragia no pós-parto; promove involução uterina; auxilia na perda de peso; e está associada ao menor risco de desenvolver diversos tipos de patologias como depres- são pós-parto, osteoporose, doenças cardiovasculares e câncer de endométrio ${ }^{12,13}$.

Economicamente, o AM gera impacto positivo no orçamento familiar, reduzindo gastos com a alimentação da criançą ${ }^{9,14}$, com consultas médicas e com a compra de medicamentos ${ }^{15}$. Quanto as despesas públicas, demonstrou-se que o aumento nas taxas de AME e/ou de AM continuado levou a uma redução nos custos com tratamento em saúde de US\$ 312 milhões nos Estados Unidos; US\$ 7,8 milhões no Reino Unido; US\$ 30 milhões na China e US\$ 1,8 milhões no Brasil ${ }^{16}$.

Outrossim, ressalta-se que a amamentação é uma prática sustentável que contribui para a proteção do meio ambiente, uma vez que o LM é um alimento naturalmente produzido e pronto para consumo, sem causar poluição, dispensando o uso de embalagens e alheio ao desperdício ${ }^{36}$.

\section{Prevalência: o que mostram os estudos?}

Números divulgados pelo Fundo das Nações Unidas para a Infância (UNICEF) em 2016 apontaram para uma expressiva prevalência do AM em todo o mundo: $44 \%$ na primeira hora de vida,
$30 \%$ de forma exclusiva em crianças menores de 6 meses e $40 \%$ de forma continuada até os 2 anos de idade ${ }^{17}$.

Victora et al.18 encontraram as maiores taxas de prevalência do AM aos 12 meses de vida na África Subsaariana, no sul da Ásia e em partes da América Latina. Contrariamente, foi inferior em países desenvolvidos, pontuando-se algumas diferenças: Reino Unido $(<1 \%)$, Suécia (16\%), Estados Unidos (27\%) e Noruega (35\%).

Diversos estudos evidenciaram a tendência de queda nas taxas de AME quanto maior a idade da criança: Polônia (1997), de $69,8 \%$ no primeiro mês de vida para $9 \%$ no sexto ${ }^{19}$; Itália, $48,7 \%$ para $43,9 \%$ aos 3 meses e 5,5\% aos 620; Espanha (Madri), $88 \%$ para $25,4 \%$ no primeiro semestre e, ainda menor aos 2 anos, 7,7\% ${ }^{21}$; México, $22,3 \%$ para $14,5 \%$ aos seis meses, sendo que na área rural o declínio foi ainda mais drástico; 36,9\% para 18,5\%22.

Apesar de possuírem características similares, pesquisas empreendidas no Haiti e na República Dominicana (RD) demonstraram tendências diferentes para a prática de AME. Na RD, verificou- 
-se queda consistente: $22,6 \%$ em 1996; $12,8 \%$ em 2002; $10,1 \%$ em 2007; e de apenas $9,5 \%$ em 2013. Já no Haiti, as taxas encontraram-se mais elevadas: $2,7 \%$ em 1994/1995; $24 \%$ em 2000; 41,6\% em 2005/2006; e 41,5\% em 2012 23 .

No Brasil, um inquérito populacional feito nas capitais e DF com menores de um ano apontou que $67,7 \%$ mamaram na primeira hora de vida e que a prevalência de AME foi de $41 \%$ em menores de 6 meses, variando desde 27,1\% em Cuibá/MT (menor) a 56,1\% em Belém/PA (maior). A duração mediana foi de 54,1 dias $(1,8$ meses) para o AME e de 341,6 dias (11,2 meses) para o $\mathrm{AM}^{37}$.

Em 2010, o MS divulgou dados sobre o AM em 227 municípios que aderiram ao levantamento realizado em 2008. Baseado nos parâmetros da OMS, verificou-se que a maioria estava em "boa situação", com prevalências entre 50-89\% de AM na primeira hora de vida. Porém, a situação do AME em menores de 6 meses foi considerada "razoável", com prevalências inferiores a $50 \%{ }^{38}$.

Uma construção de série histórica a partir de dados provenientes da Pesquisa Nacional de Saúde (PNS) - 1986, 1996, 2006 e 2013, apontou tendência crescente das taxas de AME até os 6 meses e AM até 1 ano de vida entre os anos de 1986 e 2006, estabilizando em 2013. Inversamente, o AM continuado até os 2 anos mostrou-se estável no primeiro período, aumentando em 2013. A prevalência dos indicadores, no entanto, encontrou-se aquém das recomendações ${ }^{24}$.

Portanto, é inegável que o desmame precoce ainda seja uma prática comum, apesar dos esforços e do consenso sobre a importância inquestionável do AM, principalmente no primeiro semestre de vida $^{25}$, o que dificulta o alcance da meta de prevalência de $50 \%$ estabelecida pela OMS para $2025^{26}$.

\section{Fatores que interferem no processo de desmame precoce}

Os principais fatores associados ao AM mostraram-se correlacionados às características maternas (idade, experiência na

No Brasil,

um inquérito

populacional feito

nas capitais e DF

com menores de um

ano apontou que

$67,7 \%$ mamaram na

primeira hora de vida

e que a prevalência de

AME foi de $41 \% \mathrm{em}$

menores de 6 meses,

variando desde

27,1\% em Cuibá/MT

(menor) a $56,1 \% \mathrm{em}$

Belém/PA (maior).

A duração mediana

foi de 54,1 dias $(1,8$

meses) para o $\mathrm{AME}$

e de 341,6 dias (11,2

meses) para o AM. amamentação, intercorrências na gestação, outros) e às do meio ao qual a mesma pertença, como a cultura, as relaçôes familiares e sociais e o padrão socioeconômico ${ }^{4}$.

A baixa escolaridade materna, assim como a idade e a renda foram apontadas como barreiras para a adesão e manutenção do AM: sugeriu-se que uma menor instrução pode dificultar a compreensão da importância do AME e dos benefícios do $\mathrm{LM}^{9}$; enquanto que a idade avançada e maior renda associaram-se à predisposição ao desmame precoce ${ }^{7}$.

A multiparidade mostrou-se benéfica para a prática ao passo que as experiências prévias contribuiriam com o processo de amamentação ${ }^{2,14}$.

O hábito de fumar causa menor secreção láctea e interfere de forma negativa no processo de aleitamento, prevalecendo o desmame precoce e a baixa continuidade do AM por 12 meses ou mais ${ }^{14,27}$.

O retorno ao trabalho foi considerado como uma das principais causas para a iniciação precoce da $\mathrm{AC}^{21,28}$. A não concessão do benefício da licença-maternidade aumentaria em $23 \%$ a chance de desmame precoce 26 .

A associação entre a Iniciativa Hospital Amigo da Criança (IHAC) e a diminuição da mortalidade infantil no Brasil mostrou-se positiva: entre as crianças nascidas nesse tipo de instituição, encontrou-se maior prevalência de AM na primeira hora de vida, bem como o aumento de $7,9 \%$ da taxa de AME em menores de 6 meses.

$\mathrm{O}$ uso da chupeta foi fortemente referenciado como obstáculo para a prática do AME, podendo reduzir em até $33 \%$ a sua duração ${ }^{17,29}$.

Realizar menos do que 6 consultas de pré-natal aumenta em 2,76 vezes a chance de amamentar por menos tempo ${ }^{30}$. Assim, tem-se nessa assistência um fator protetivo para o AME, sendo o momento oportuno para orientações quanto à prática, seus benefícios e manejo das dificuldades que possam ser vivenciadas ${ }^{14,21}$.

A família, sobretudo o parceiro e a mãe da nutriz, exercem influência ao apoiar, ou não, a prática da amamentação; quando positiva, colabora com o sucesso do $\mathrm{AM}^{12,31}$. 


\section{artigo}

Araújo, T.P.; Formigosa, L.A.C.; Maciel, A.P.

Prevalência e fatores associados ao desmame precoce: uma revisão integrativa

"Leite fraco" ou "só o leite não sustenta"; "criança que nasceu prematura ou com baixo peso não deve mamar no peito"; "rachaduras no bico do seio"; "empedramento do leite"; "mastite e/ou abscesso"; "o leite está secando"; "não querer amamentar"; "problemas no mamilo"; "dor ao amamentar"; e "bebê sente cólicas quando mama" foram motivos apontados para não aderir ao $\mathrm{AME}^{32}$.

\section{Introdução alimentar precoce}

Assim como a manutenção do AM é imprescindível, a introdução de alimentos seguros, acessíveis e culturalmente aceitos na dieta da criança, em época oportuna e de forma adequada, torna-se indispensável para a promoção do desenvolvimento saudável e prevenção de distúrbios nutricionais de grande impacto na Saúde Pública ${ }^{30}$

Conforme o "Guia Alimentar" 35 , nenhum outro tipo de alimento necessita ser dado ao bebê enquanto estiver em amamentação exclusiva. A partir dos 6 meses, outros alimentos devem fazer parte das refeições da criança, especialmente in natura ou minimamente processados. A realidade, porém, mostrou-se aquém das recomendações.

O leite foi apontado como principal alimento introduzido precocemen- te $(79,3 \%)$, seguido de massas/mingaus $(58,7 \%)$ e das frutas/vitaminas $(11,1 \%)$. $\mathrm{O}$ consumo lácteo iniciou entre 1 e 4 meses de idade (66\%); sendo que $51,6 \%$ optaram pelas fórmulas infantis, cuja média de uso foi de 1,5 mês, posteriormente substituídas pelos leites integrais ${ }^{33}$.

Lopes et al. ${ }^{1}$, através de entrevista domiciliar com os responsáveis de 545 crianças menores de 24 meses residentes na zona urbana de uma capital brasileira, evidenciaram que aos 180 dias de vida, apenas $4 \%$ das crianças estavam em AME e $43,4 \%$ já haviam iniciado $\mathrm{AC}$; aos 3 meses de idade, $56,8 \%$ dos lactentes já recebiam água; $15,5 \%$, suco de fruta natural/fórmula infantil; e 10,6\%, leite de vaca. Antes do primeiro ano, quase metade já fazia ingestão de doces; $25 \%$, macarrão instantâneo; e $30 \%$, açúcar e achocolatado.

$\mathrm{O}$ início inadequado da AC mostrou-se associado, principalmente, com a falta de conhecimento das mães quanto aos passos que compóem a prática e com a crença e cultura perpassadas pelos familiares $^{28}$. Quanto à orientação recebida por mães de crianças com idade entre 4 e 24 meses, estudo observou que 62,2\% havia sido por parte de pediatras e/ou outro profissional da saúde; $19,6 \%$, por nutricionistas ${ }^{34}$.

\section{CONCLUSÃO}

Apesar de ser um ato natural, o AM é permeado por fatores que o tornam de responsabilidade não só da mulher que amamenta, mas de toda a sociedade, que deve atuar como rede de apoio e orientação.

Embora os benefícios do LM sejam amplamente divulgados e a prática amparada por movimentos políticos e sociais, observa-se que, mundialmente, as taxas de adesão encontram-se distantes da meta proposta pela OMS, contribuindo para a ocorrência de mortes preveníveis entre crianças na primeira infância.

Nesse cenário, faz-se necessário adequar e fortalecer a escuta profissional no sentido de prestar atendimento que acolha as dúvidas, os medos e as incertezas moldadas pelo ambiente no qual as gestantes e nutrizes estão inseridas.

Ademais, é importante considerar que as práticas alimentares também são fruto da cultura e valores adotados. Portanto, contemplar as diferenças regionais e empreender estudos para além das regiões metropolitanas poderia contribuir para a elaboração de estratégias mais efetivas em relação ao incentivo à amamentação e à introdução de uma AC saudável.

\section{REFERÊNCIAS}

1. Lopes WC et al. Alimentação de crianças nos primeiros dois anos de vida. Rev Paul Pediatr. 2018;36(2): 164-170.

2. Ferreira HLOC, Oliveira MF, Bernardo EBR, Almeida PC, Aquino PS, Pinheiro AKB. Fatores associados à adesão ao aleitamento materno exclusivo. Ciên Saúde Colet. 2018;23(3):683-690.

3. Brasil. Ministério da Saúde. Secretaria de Atenção Primária à Saúde. Departamento de Atenção Básica. Saúde da Criança: Aleitamento Materno e Alimentação Complementar. Cadernos de Atenção Básica, n. 23. 2a. ed. Brasília: Ministério da Saúde; 2015. $184 \mathrm{p}$

4. Pivetta HMF et al. Prevalência de aleitamento materno e fatores associados: uma revisão de literatura. Rev Ciênc Méd Biol. 2018;17(1):95-101.

5. Souza MT, Silva MD, Carvalho R. Revisão integrativa: o que é e como fazer. Einstein. 2010 mar.;8(1):102-106.

6. Costa FS, Silva JLL, Machado EA, Soares LM, Brezolin CA, Silva JVL. Promoção do aleitamento materno no contexto da Estraté- gia de Saúde da Família. Rev Rede cuid saúde. 2019 jul.;13(1):4458.

7. Oliveira DS, Boccolini CS, Faerstein E, Verly-Jr E. Breastfeeding duration and associated factors between 1960 and 2000. J Pediatr. 2017;3(2):130-135.

8. Walters DD, Phan LTH, Mathisen R. The cost of not breastfeeding: global results from a new tool. Health policy plan. 2019;34(6):407-417.

9. Margotti E, Margotti W. Fatores relacionados ao aleitamento materno exclusivo em bebês nascidos em Hospital Amigo da Criança em uma capital do Norte brasileiro. Saúde Debate. 2017;41(114):860-871.

10. José DKB, Vitiato JA, Hass K, França TCS, Cavagnari MAV. Relação entre desmame precoce e alergias alimentares. Visão Acadêmica. 2016;17(3):66-74.

11. Carvalho-Ramos II, Duarte RTD, Brandt KG, Martinez MB, Taddei CR. Breastfeeding increases microbial community resil- 
ience. J Pediatr. 2018;94(3): 258-267.

12. Tessari W, Soares LG, Soares LG, Abreu IS. Percepção de mães e pais adolescentes sobre 0 aleitamento materno. Enferm Foco. 2019;10(2):83-89.

13. Del Ciampo LA, Del Ciampo IRL. Breastfeeding and the benefits of lactation for women's health. Rev Bras Ginecol Obst. 2018;40(6):354-359.

14. Santana GS, Giugliani ERJ, Vieira TO, Vieira GO. Factors associated with breastfeeding maintenance for 12 months or more: a systematic review. J Pediatr. 2018;94(2):104-122.

15. Nunes LM. Importância do aleitamento materno na atualidade. Bol Cient Pediatr. 2015;4(3):55-58.

16. Rollins NC et al. Why invest, and what it will take to improve breastfeeding practices? Lancet. 2016;387(10017):491-504.

17. Silva VAAL, Caminha MFC, Silva SL, Serva VMSBD, Azevedo PTACC, Batista Filho M. Maternal breastfeeding: indicators and factors associated with exclusive breastfeeding in a subnormal urban cluster assisted by the Family Health Strategy. J Pediatr. 2019;95(3):298-305.

18. Victora CG et al. Breastfeeding in the 21st century: epidemiology, mechanisms and lifelong effect. Lancet. 2016;387(10017):475-490.

19. Zielińska MA, Sobczak A, Hamułka J. Breastfeeding knowledge and exclusive breastfeeding of infants in first six months of life. Rocz Panstw Zakl Hig. 2017;68(1):51-59.

20. Mosca $\mathrm{F}$ et al. Determinants of breastfeeding discontinuation in an Italian cohort of mother-infant dyads in the first six months of life: a randomized controlled trial. Ital $\mathrm{j}$ pediatr. 2018;44(1):134-144.

21. Ramiro González MD et al. Prevalencia de la lactancia materna y factores asociados con el inicio y la duración de la lactancia materna exclusiva en la Comunidad de Madrid entre los participantes en el estudio ELOIN. An pediatr. 2018;89(1):32-43.

22. Mota-Castilho PJ, Hernández-Ibarra LE, Pelcastre-Villafuente $\mathrm{BE}$, Rangel-Flores $\mathrm{YY}$. Experiencias y creencias de madre sobre la lactancia materna exclusiva en una región de México. J nurs health. 2019;9(1):e199103.

23. Mclennan JD. Changes over time in early complementary feeding of breastfeed infants on the island of Hispaniola. Rev Panam Salud Pública. 2018 jun.;41(26):1-8.

24. Boccolini CS, Boccolini PMM, Monteiro FR, Venâncio SI, Giugliani ERJ. Tendência de indicadores do aleitamento materno no Brasil em três décadas. Rev Saúde Pública [internet]. 2017 [cited 2020 Jan 18];51:108. Available from: https://doi.org/10.11606/s $1518-8787.2017051000029$

25. Santos PV, Carvalho e Martins MC, Tapety FI, Paiva AA, Fonseca FMNS, Brito AKS. Desmame precoce em crianças atendidas na Estratégia Saúde da Família. Rev Eletr Enf. [Internet]. 2018 [cited 2019 Dec 15];20:v20a05. Available from: http://doi. org/10.5216/ree. v20.43690.
26. Monteiro FR, Buccini GS, Venâncio SI, Costa THM. Influence of maternity leave on exclusive breastfeeding. J Pediatr. 2017;93(5):475-481.

27. Schincaglia RM, Oliveira AC, Sousa LM, Martins KA. Práticas alimentares e fatores associados à introdução precoce da alimentação complementar entre crianças menores de seis meses na região noroeste de Goiânia. Epidemiol Serv Saúde. 2015;24(3):465-474.

28. Mariano BS, Moraes JL, Ravazzani EDA. Saberes maternos acerca da alimentação complementar. Cad da Esc de Saúde. 2017;17(1):30-41.

29. Mosquera PS et al. Factors affecting exclusive breastfeeding in the first month of life among Amazonian children. PLOS ONE. 2019;14(7):e0219801.

30. Mendes SC, Lobo IKV, Sousa SQ, Vianna RPT. Fatores relacionados com uma menor duração total do aleitamento materno. Ciên Saúde Colet. 2018;24(5):1821-1829.

31. Nóbrega VCF, Melo RHV, Diniz ALTM, Vilar RLA. As redes sociais de apoio para o Aleitamento Materno: uma pesquisa-ação. Saúde Debate. 2019 abr.-jun.;43(121):429-440.

32. Borges NR, Sousa DKS, Pereira RJ, Castro JGD. Caracterização e prevalência do aleitamento materno em uma população atendida na rede pública de saúde de Palmas/TO, Brasil. Rev Bras Pesq Saúde. 2016;18(4):30-

33. Albuquerque AO, Dantas KB, Tomé MABG, Aire JS, Gubert FA, Martins MC. Hábitos alimentares de crianças com até 6 meses em alimentação complementar e/ou desmame precoce. Enferm atual. [Internet]. 2018 [cited 2019 Dec 18];86(24). Available from: http://revistaenfermagematual.com/ arquivos/ED_86_ REVISTA_24/30.pdf.

34. Giesta JM, Zoche E, Corrêa RS, Bosa VL. Fatores associados à introdução precoce de alimentos ultraprocessados na alimentação de crianças menores de dois anos. Ciên Saúde Colet. 2019;24(7):2387-2397.

35. Brasil. Ministério da Saúde. Secretaria de Atenção Primária à Saúde. Departamento de Promoção da Saúde. Guia alimentar para crianças brasileiras menores de dois anos. Brasília: Ministério da Saúde; 2019. 265 p.

36. Brasil. Ministério da Saúde. Secretaria de Atenção à Saúde. Departamento de Ações Programáticas Estratégicas. Bases para a discussão da Política Nacional de Promoção, Proteção e Apoio ao Aleitamento Materno. Brasília: Ministério da Saúde; 2017. 68 p.

37. Brasil. Ministério da Saúde. Secretaria de Atenção à Saúde. Departamento de Ações Programáticas e Estratégicas. II Pesquisa de Prevalência de Aleitamento Materno nas Capitais Brasileiras e Distrito Federal. Brasília: Ministério da Saúde; 2009. 108 p.

38. Brasil. Ministério da Saúde. Secretaria de Atenção à Saúde. Departamento de Ações Programáticas e Estratégicas. Pesquisa de Prevalência de Aleitamento Materno em Municípios Brasileiros. Brasília: Ministério da Saúde; 2010. 63 p. 